Veritas Et Scientia

Vol. 7, N 2, 941-952

Julio - Diciembre del 2018.

ISSN Edición Online: 2617-0639

https://doi.org/10.47796/ves.v7i2.71

\title{
EFICACIA DE LAS MEDIDAS DE PROTECCIÓN EN PROCESOS DE VIOLENCIA FAMILIAR GARANTIZAN EL DERECHO A LA INTEGRIDAD DE VÍCTIMAS EN LA CORTE SUPERIOR DE JUSTICIA DE TACNA, SEDE CENTRAL, 2017.
}

\author{
EFFECTIVENESS OF PROTECTIVE MEASURES IN FAMILY VIOLENCE PROCESSES \\ GUARANTEE THE RIGHT TO INTEGRITY OF VICTIMS IN THE SUPERIOR COURT OF \\ JUSTICE OF TACNA, HEADQUARTERS, 2017.
}

\author{
Ada Paola Mejía Rodríguez ${ }^{1}$ \\ Presentado: $18 / 08 / 218$ \\ Aceptado: $17 / 09 / 2018$ \\ Publicado online:27/12/2018
}

\footnotetext{
${ }^{1}$ Magister en Derecho
}

\section{RESUMEN}

La investigación realizada es de corte jurídico social, de tipo descriptivoexploratorio y dogmático-jurídico. Se aplicaron cuestionarios anónimos (17 preguntas con alternativa múltiple y de tipo dicotómico) a 341 participantes, entre los cuales se incluyen abogados litigantes en procesos judiciales de violencia familiar, defensores públicos, magistrados y secretarios de juzgados de familia, abogados del Centro de Emergencia Mujer (Tacna) y fiscales de familia (provinciales y adjuntos). Asimismo, se aplicó una ficha de análisis documental para recoger datos de 338 resoluciones de audiencias de medidas de protección en procesos de violencia familiar. La investigación pretendió determinar la eficacia de las medidas de protección otorgadas a las víctimas que denuncian violencia familiar ante juzgados de familia de la Corte Superior de Justicia de Tacna (CSJT). En base a los resultados de la encuesta realizada y a la revisión y análisis de las resoluciones judiciales, se llegó a la conclusión que son eficaces las medidas de protección otorgadas a las víctimas por los magistrados de los cuatro Juzgados de Familia de la CSJT ya que garantizan el derecho a la integridad de las víctimas. Asimismo, si bien la propia ley expresa taxativamente que es la Policía Nacional del Perú la entidad responsable de ejecutar las medidas de protección dictadas por el juzgado de familia correspondiente, la mayoría de los encuestados considera que si existe una supervisión eficaz del cumplimiento de las medidas de protección, lo que significa que si se realiza un seguimiento de manera periódica de dichas medidas otorgadas a favor de la víctima. Por otro lado, la percepción que tienen los operadores de justicia encuestados es que la ley 30364 y la no regulación de la conciliación resulta más efectiva 
que la ley 26260, ya que la ley 30364 garantiza el derecho a la integridad de la víctima, genera más confianza en la justicia y los jueces y la proyecta como una medida más severa a la sociedad.

Palabras clave: violencia familiar, medidas de protección, Juzgados de familia

\begin{abstract}
The research carried out is of a social legal nature, of a descriptiveexploratory and dogmatic-juridical nature. Anonymous questionnaires (17 multiple choice and dichotomous questions) were applied to 341 participants, including trial lawyers in family violence, public defenders, magistrates and family court clerks, lawyers for the Women's Emergency Center (Tacna) and family prosecutors (holders and deputies). Likewise, a document analysis sheet was applied to collect data on 338 resolutions of hearings on protection measures in family violence processes. The investigation sought to determine the effectiveness of the protection measures granted to victims who denounce family violence before family courts of the Superior Court of Justice of Tacna (CSJT). Based on the results of the survey conducted and the review and analysis of the judicial resolutions, it was concluded that the protection measures granted to the victims by the magistrates of the four Family Courts of the CSJT are effective since guarantee the right to the integrity of the victims. Likewise, although the law itself expressly states that the National Police of Peru is the entity responsible for executing the protective measures ordered by the corresponding family court, most of the respondents consider that there is effective supervision of compliance with the measures of protection, this means that there is periodic follow-up of said measures granted in favor of the victim. On the other hand, the perception of the surveyed justice operators is that Law 30364 and non-regulation of conciliation is more effective than Law 26260, since Law 30364 guarantees the victim's right to integrity, generates more trust in justice and judges and projects it as a more severe measure to society.
\end{abstract}

Keywords: family violence, protection measures, Family courts.

\title{
INTRODUCCION
}

La ley 30364 ha sido creada con miras a la prevención, erradicación y sanción de toda forma de violencia ejercida en el ámbito público o privado contra de las mujeres por su condición de tales, y contra los integrantes del grupo familiar, denominación que hace clara distinción con la ley que la precedía, la misma que abarcaba la violencia, pero en un ámbito más general, es decir una ley de protección frente a la violencia familiar en general. Asimismo, esta nueva norma incorporada al marco legal peruano se hace vigente desde el mes de noviembre del 2015, la misma que incorpora en comparación de la ley 26260, que solo regulaba la violencia familiar física y psicológica, pues añade la violencia sexual y económica o patrimonial dentro de los tipos de violencia regulados.

Aunado a ello, la nueva ley trae consigo un cambio sustancial en la etapa de protección, la misma que se llevará a cabo ante un juez de familia para la expedición o no de las medidas de protección a favor de la víctima de violencia familiar, lo que antes era expedido por el fiscal de 
familia, además que en la misma audiencia de medidas de protección se podrá solicitar las medidas cautelares de tenencia provisional, alimentos, y régimen de visitas, lo cual aparentemente suena como grandes cambios positivos, pero resulta ser confrontado con la realidad, por lo que esta investigación tiene como finalidad mostrar los impactos de la ley 30364 en la sociedad tacneña, en cuanto a la aplicación de ley 30364, y que tan eficaz resulta ser a favor de la víctima de violencia familiar en la etapa de protección. Se ha querido aplicar ello en los expedientes judiciales expedidos en el año 2017 en los Juzgado de Familia del Cercado de Tacna, y las encuestas a los operadores de justicia, es decir, jueces de familia, fiscales de familia, abogados litigantes, abogados del Centro de Emergencia Mujer, defensores públicos, y personal fiscal y judicial.

La violencia contra la mujer y los integrantes del grupo familiar es un problema que el Estado busca combatir y erradicar, ya que es una de las caras más dolorosas de nuestro país. Sin duda, un problema social con amplias dimensiones que atraviesan familias de distintos estratos sociales, culturales, de educación, religión, raza, etnia y edad, producto de desigualdades por la aparente dominación/subordinación entre agresor y víctima, e incluso cuestiones que van más allá como celos, envidia, bajos recursos económicos, socioculturales, entre otros, los mismos que responden a patrones sociales y culturales profundamente arraigados en la sociedad que a pesar de los años se mantienen como prácticas del quehacer cotidiano de algunos agresores sobre sus víctimas. Según la encuesta realizada el año 2015 por el Instituto Nacional de Estadística e Informática (INEI), el mismo que tiene como finalidad determinar la violencia física ejercida por departamentos, la mayor tasa de prevalencia de violencia la lleva Apurímac en $49,5 \%$, Cuzco con $44.7 \%$, Arequipa con $44,4 \%$, para luego de ello colocarnos en el décimo lugar a Tacna con un $35,2 \%$ del total de la población nacional respecto a las mujeres que han vivido un evento de violencia familiar; de los cuales, la mayor parte afectada suele ser mujeres que conforman o conformaban una familia. Sin duda estamos vulnerables a seguir un proceso por violencia familiar. La ley 30364 y su reglamento plantean como medida para prevenir la violencia familiar, las bien llamadas "Medidas de protección" que tienen como objeto que el Juez de Familia con su potestad discrecional y en merito a los primeros actuados de la Policía Nacional o el Ministerio Publico pueda dictar medidas de protección a las víctimas a efectos que el agresor no pueda ejercer actos vulneratorios del derecho a la integridad física, psicológica, sexual o económica de las víctimas, ya que el devenir en los mismos actos acarrearía una denuncia por el delito de Resistencia o Desobediencia a la autoridad, con penas reguladas en el Código Penal.

En atención de los diversos estudios realizados en mérito a la incidencia de la violencia familiar en nuestro país, es claro que es un fenómeno agobiante dado que daña una de las esferas más preciadas por el Derecho, la cual es la vida e integridad física de las víctimas, la misma que se ve mermada por la cada vez más preocupante cifra de agresiones y muerte de las víctimas en el día a día y de las constantes campañas y luchas televisivas por buscar una igualdad entre la mujer y el sexo opuesto, aunado a ello la incorporación de una Ley que busca que las medidas de protección sean impuestas ahora por un Juez de Familia y ya no por el Representante del Ministerio Público. Dichas medidas son ejecutadas y supervisadas por la Policía Nacional, lo cual aparentemente suena positivo, pero resulta difícil comprobarlo en la práctica, por lo que nos es de vital importancia conocer el impacto de la nueva ley y si las medidas de protección son eficaces en cuanto a la lucha contra la violencia familiar en el marco de la ley 30364.

Cifuentes (2009) sustentó la persistencia de distinciones legales anacrónicas e injustificadas basadas en el género, contraviene el objeto y la finalidad de los compromisos positivos adoptados por el Estado de Guatemala en relación a la violencia intrafamiliar en el municipio de San Juan Sacatepéquez, departamento de Guatemala, debido a que no aplica la justicia ni se toman medidas concretas. Morales (2010) en Chile refiere que las lesiones son el delito más 
común dentro de los ilícitos cometidos en contexto de violencia intrafamiliar, de ahí que resulte relevante saber cómo influye este contexto al juzgarlas. Hernández (2014) en la Universidad de Sevilla (España), propone que actualmente el problema de la violencia intrafamiliar está más visibilizado y ello ha traído como consecuencia la expedición de la Ley Orgánica de Protección Integral a la Violencia de Género. Entrando en el mismo tema, Vera (2014) en Ecuador afirma que a partir de la entrada en vigencia de la Ley 103 que toda mujer que sea sometida a amenazas, violencia física, moral, psicológica o que sea objeto de acceso carnal violento por parte de quien haga vida marital con ella, debe conocer de la existencia de este instrumento jurídico especial que la protege de manera directa y real. La Ley 103 abarca la protección de diferentes derechos y principios que deberán ser respetados y proporcionan rapidez y seriedad en el asunto. Condori (2016) sustentó en Puno que el impacto de la ley 30364 en términos generales es positivo en el Centro de Emergencia Mujer de la Provincia de llave, asimismo, el tipo de violencia que mayormente sufren las mujeres en Puno es la violencia física y psicológica y el $98 \%$ de ellas han solicitado patrocinio legal del Centro Emergencia Mujer. Ventura (2016) en Huánuco concluye que el proceso por violencia familiar (vigente en esa época a través de la ley $\mathrm{N}^{\circ} 29282$ ) no es eficaz, puesto que no contiene mecanismos efectivos de protección para las víctimas, ni garantizan totalmente los derechos de las víctimas de violencia de género. Orna (2013) en el distrito de San Juan de Lurigancho (Lima), logró comprobar que la violencia familiar es un fenómeno social que revela que las víctimas de la violencia familiar son principalmente las mujeres en proporción de 9 a 1 comparado con la violencia hacia los hombres. Chávarri (2015) en la Demuna del distrito de Yonan - provincia de Contumaza refiere que las mujeres víctimas dependen económicamente de sus parejas; y por ello se vuelven más vulnerables a los maltratos del agresor, quien es su pareja. Arriola (2013), en que los juicios de agresión familiar duran demasiado, y que esto validó al hecho que, al momento que se investigaron los acontecimientos que sirvieron de explicaciones, no se contaba con una directriz u observadora de valoración del mismo. Rodriguez (2013) también en Tacna, encontró que existe relación significativa entre el tratamiento de las medidas de protección dictadas por el Ministerio Público y la violencia familiar entre cónyuges y convivientes en el distrito judicial de Tacna. Díaz (2010) afirma que las medidas de protección que establece la ley frente a la violencia familiar están sólo en papel y no se cumplen en la práctica, por cuanto muchas veces, pese a que están dictadas por un órgano Jurisdiccional, y que por tanto deberían cumplirse obligatoriamente, no es realmente así.

Derechos fundamentales de la víctima en la ley 30364 propugna el Derecho a una vida libre de violencia, Derecho a la asistencia y a la protección integral, Acceso a la información, Asistencia Jurídica y Defensa Pública, Promoción, Prevención y Atención de Salud y Atención Social. El Estado brinda programas sociales a las víctimas de violencia, garantizando la confidencialidad y brindando trato digno, respetando sus derechos, no permitiendo la revictimización, creyendo en la veracidad de la denuncia presentada. Según Junco, J $(2017$, p.2) refiere que siempre han existido los famosos mitos acerca del por qué se ejecutan actos de violencia contra la víctima. Es así que Lorente Acosta (2005) habla de "los hombres agresores e indica de manera clara que no es posible definir, con la precisión que requieren esquemas criminológicos tradicionales, el perfil de una personalidad masculina violencia". Según Loli (2015), el enfoque de género permite visualizar y reconocer la existencia de relaciones de jerarquía y desigualdad entre hombres y mujeres. Ello implica reconocer que en actos de violencia familiar existen relaciones asimétricas entre hombres y mujeres. De otro lado, Oxfam (2014) refiere "Aunque todas las mujeres de una u otra manera sufren discriminación de género, no todas la sufren del mismo modo; existen otros factores que se combinan para determinar la posición social de una mujer o de un hombre: raza, color de piel, edad, etnicidad, idioma, ascendencia, orientación sexual, 
religión, clase socio-económica, cultural, capacidad, localización geográfica, estatus como migrante, persona que vive con $\mathrm{VIH} /$ Sida".

\section{Objetivos}

a. Determinar si los mecanismos jurídicos de supervisión son eficaces (fiscalizan y monitorean) en la ejecución de las medidas de protección.

b. Precisar si las medidas de protección impuestas por los jueces en procesos de violencia familiar a las victimas disuaden a los agresores a no reincidir en actos violentos.

\section{METOdOLOGÍA}

Investigación de tipo cualitativa-cuantitativa, socio-jurídica, de diseño de investigación es exploratorio, descriptiva y dogmático-jurídico. Se llevó a cabo en los Juzgados de Familia de la Corte Superior de Justicia de Tacna (CSJT) desde el mes de enero del año 2017 hasta el mes de diciembre del año 2017. Las unidades de estudio fueron los Expedientes judiciales y Abogados litigantes, Abogados de la Defensoría Pública, Abogados del Centro de Emergencia Mujer, Fiscales de Familia y sus Asistentes en función fiscal, Jueces de Familia, y sus Secretarios y Asistentes de los Juzgados de Familia. El tamaño de la muestra fueron 338 resoluciones de audiencias de otorgamiento de medidas de protección devenidas de denuncias por violencia familiar: 103 de ellos corresponde al $1^{\circ}$ Juzgado de Familia, 100 de ellos al $2^{\circ}$ Juzgado de Familia, 60 al 3o Juzgado de Familia y 75 pertenece al 40 Juzgado de Familia. Adicionalmente 341 participantes: 304 abogados litigantes,09 abogados del Centro de Emergencia Mujer, 04 abogados de la Defensoría Pública,08 sujetos entre ellos 04 Fiscales de Familia y sus 04 asistentes, 16 sujetos entre ellos 04 Jueces de Familia, y sus 04 Secretarios y 04 Asistentes de los Juzgados de Familia. Se realizaron 3 tipos de procedimientos de investigación: técnica de encuestas, técnica de observación y técnica de análisis documental o bibliográfica.

\section{RESULTADOS}

En cuanto a los datos de abogados encuestados por el lugar donde desempeñan sus labores de experiencia en la rama de Familia, se encontró que el $89,15 \%$ de la muestra son abogados litigantes en la rama de Familia, además que el $2,64 \%$ de la muestra son abogados que trabajan en el Centro de Emergencia Mujer. Asimismo, el 2,35\% de la muestra son abogados entre fiscales y sus asistentes en función fiscal que laboran en la Fiscalía de Familia sede Central. Así también se tiene que el $4,69 \%$ de la muestra son abogados entre jueces, y sus especialistas y asistentes de los Juzgados de Familia sede Central. Finalmente, el 1,17\% de la muestra son Defensores Públicos en el Área de Familia. Respecto al tiempo de experiencia en la rama de Familia, se encontró que el $37,54 \%$ de la muestra tienen entre 1 a 5 años de experiencia, además que el 35,19\% de la muestra tienen entre 6 a 10 años de experiencia, asimismo, el $17,01 \%$ de la muestra tienen entre 11 a 15 años de experiencia y finalmente el $10,26 \%$ de la muestra tienen entre 16 a 20 años de experiencia.

\section{Tabla 01}

Respuesta a cuestionario aplicado

\begin{tabular}{llc}
\hline & Si (\%) & No (\%) \\
\hline Existen mecanismo de supervisión de ejecución de medidas de protección & 79,77 & 20,23 \\
Los mecanismos de supervisión de medidas de protección son eficaces & 52,79 & 47,21 \\
Si el cumplimiento de las medidas de protección se realiza a través de un & 52,79 & 47,21 \\
seguimiento de manera periódica por parte de la Policía Nacional &
\end{tabular}


El no seguimiento del cumplimiento de la medida de protección de manera periódica puede provocar la incursión de nuevo acto de violencia familiar

El no seguimiento del cumplimiento de la medida de protección de manera periódica vulneraria el derecho a la integridad de la víctima al ponerla en riesgo

La Policía Nacional debe seguir siendo normativamente quien ejecute el cumplimiento de las medidas de protección

Las medidas de protección deben ser dictadas por jueces de familia como lo refiere la ley 30364, o deben seguir siendo dictadas por los fiscales de familia como en la ley 26260

El otorgamiento de medidas de protección por un juez genera más eficacia que la dictada por un fiscal

El otorgamiento de medidas de protección por un juez genera más eficacia para no reincidir nuevamente en violencia familiar en la victima

El apercibimiento de ser denunciado por el Delito de Resistencia y Desobediencia a la Autoridad, si el agresor incumple con la medida de protección dicta, resulta eficaz para garantizar el derecho a la integridad sobre la victima

La ejecución de medidas de protección en casos de Violencia Familiar son más eficaces según la ley 30364 en comparación con la ley 26260

$69,5 \quad 30,5$

La no regulación de la conciliación en procesos de violencia familiar genera en las victimas más confianza en la justicia y en los jueces en comparación con la ley 26260

La no regulación de la conciliación como medio de solución de procesos de violencia familiar la proyecta como una medida más severa frente al agresor y la sociedad

La no regulación de la conciliación permite que la víctima se sienta más empoderada en un proceso de violencia familiar frente a su agresor

La no regulación de la conciliación permite que la víctima se sienta más protegida en cuanto a su derecho a la integridad respecto a la violencia familiar ejercida por su agresor

Se dictó medidas de protección a favor de la víctima

Se presentó casos de reincidencia en los procesos de violencia familiar denunciados

$\begin{array}{rr}63,93 & 36,07 \\ 63,93 & 36,07 \\ 74,49 & 25,51 \\ 93,2 & 6,8 \\ 2,66 & 97,34\end{array}$

En cuanto a los datos de expedientes judiciales sobre violencia familiar con la aplicación de la ley 30364, se encontró que el $30,47 \%$ de la muestra pertenecen al Primer Juzgado de Familia, además que el $29,59 \%$ de la muestra pertenece al Segundo Juzgado de Familia, asimismo el $17,75 \%$ de la muestra pertenece al Tercer Juzgado de Familia y finalmente, el $22,19 \%$ de la muestra pertenece al Cuarto Juzgado de Familia de la Sede Central de la Corte Superior de Justicia de Tacna año 2017.

Respecto a comisaría de donde se presentó la denuncia por violencia familiar, un porcentaje mayoritario lo tiene la Comisaría de Gregorio Albarracín Lanchipa. Seguido por las Comisarías Central, De la Familia, Vigil, Leguía, Natividad y Pocollay que presentan denuncias pertenecientes a su sector considerables actos de violencia y finalmente, las Comisarías de Boca del Río, Calana, Sama Inclán, Pachía e Ite donde es escasa la presencia de denuncias por violencia familiar,

En cuanto a los datos de expedientes judiciales sobre violencia familiar con la aplicación de la ley 30364 , encontramos el $14,2 \%$ de la muestra perteneciente a la violencia física, además un $39,05 \%$ de la muestra pertenece violencia psicológica, luego un $1,18 \%$ de la muestra corresponde a la violencia sexual, seguida del 0,9\% por violencia sexual; así también 
encontramos que el $44,08 \%$ de la muestra pertenece a la violencia física y psicológica, finalmente el $0,59 \%$ pertenece a la violencia sexual y psicológica (Tabla 02)

En cuanto a los datos del genero de las víctimas de violencia familiar encontramos que el $82,29 \%$ de la muestra pertenece al género femenino, mientras que un $17.71 \%$ de la muestra representa al género masculino. (Tabla 02)

En cuanto a los datos del genero de los agresores (Tabla 03) de violencia familiar en los expedientes judiciales sobre violencia familiar con la aplicación de la ley 30364, encontramos e1 $81,56 \%$ de la muestra pertenece al género masculino, mientras que un $18,44 \%$ de la muestra representa al género femenino.

Tabla 02

Tipo de violencia denunciada por la victima

\begin{tabular}{lcc}
\hline \multicolumn{1}{c}{ Opciones } & $\mathrm{F}$ & $\%$ \\
\hline Física & 48 & 14,2 \\
Psicológica & 132 & 39,05 \\
Sexual & 4 & 1,18 \\
Patrimonial & 3 & 0,9 \\
Física y Psicológica & 149 & 44,08 \\
Sexual y Psicológica & 2 & 0,59 \\
\hline \multicolumn{1}{r}{ Total } & 338 & $100 \%$
\end{tabular}

Fuente: Expedientes judiciales de violencia familiar con la aplicación de la ley 30364 en los Juzgados de Familia de la Corte Superior de Justicia de Tacna, Sede Central.

Tabla 03

Género del (s) Agresor (es) de Violencia Familiar

\begin{tabular}{cccc}
\hline & Opciones & $\mathrm{F}$ & $\%$ \\
\hline Femenino & 66 & 18,44 \\
Masculino & & 292 & 81,56 \\
\hline & Total & 358 & $100 \%$
\end{tabular}

Fuente: Expedientes judiciales de violencia familiar con la aplicación de la ley 30364 en los Juzgados de Familia de la Corte Superior de Justicia de Tacna, Sede Central.

En cuanto a los datos del vínculo entre víctimas y agresores de violencia familiar, encontramos que e1 $20,71 \%$ de la muestra pertenece a los convivientes, mientras que un $17,98 \%$ de la muestra representa a los ex convivientes, un $15,80 \%$ de la muestra no señala vinculo; también el $10,63 \%$ de la muestra pertenece a padres-hijos, además de un $9,81 \%$ de la muestra a cónyuges. De otro lado encontramos, un $4,09 \%$ de la muestra a ex cónyuges y un $3,54 \%$ de la muestra tanto a hermanos como también tíos-sobrinos, para luego encontrar un $3,27 \%$ a cuñados y un $2,45 \%$ de la muestra a ex parejas o ex enamorados. Finalmente encontramos a un $1,63 \%$ de la muestra tanto para parejas o enamorados como también para suegros-nueros, también observamos que un $1,36 \%$ de la muestra es entre padrastros-hijastros, al igual que un $1,09 \%$ de la muestra tanto a primos como a abuelos-nietos, un $0,82 \%$ sin vínculo alguno y un $0,54 \%$ a hermanastros.

Respecto a presencia del agresor y víctima en audiencia de medidas de protección, se encontró que el 39,94 \% de la muestra no se aprecia presencia del agresor ni víctima en la audiencia, 
mientras que un $31,36 \%$ de la muestra representa a la presencia de víctima y agresor, además un $15,98 \%$ solo se encuentra la victima presente en la audiencia, además un $8,28 \%$ solo se encuentra al agresor en la audiencia, y finalmente un $4,44 \%$ no se realizó la audiencia.

\section{Tabla 04}

Tipo de medidas de protección dictadas a favor de la víctima de violencia familiar

\begin{tabular}{|c|c|c|c|}
\hline & Opciones & $f(n=338)$ & $\%$ \\
\hline (1) & Retiro del agresor del domicilio & 20 & 2,28 \\
\hline (2) & $\begin{array}{l}\text { Impedimento de acercamiento o proximidad a la víctima } \\
\text { a la distancia de } 300 \mathrm{mts} \text {. }\end{array}$ & 271 & 30,83 \\
\hline (3) & $\begin{array}{l}\text { Prohibición de comunicación con la victima vía epistolar, } \\
\text { telefónica, electrónica; asimismo, vía chat, redes } \\
\text { sociales, red institucional, intranet u otras redes o } \\
\text { formas de comunicación. }\end{array}$ & 14 & 1,59 \\
\hline (4) & $\begin{array}{l}\text { Prohibición del derecho de tenencia y porte de armas } \\
\text { para el agresor. }\end{array}$ & 0 & 0 \\
\hline (5) & Inventario sobre sus bienes & 0 & 0 \\
\hline (6) & $\begin{array}{l}\text { Cualquier otra requerida para la protección de la } \\
\text { integridad personal y la vida de sus víctimas o } \\
\text { familiares (visita social a efectos de conocer situación } \\
\text { socio familiar) }\end{array}$ & 22 & 2,5 \\
\hline & Prohibición de incurrir en nuevos actos de violencia & 315 & 35,84 \\
\hline & Evaluación y tratamiento de víctima y agresor & 214 & 24,35 \\
\hline & $\begin{array}{l}\text { Se declaró NO HA LUGAR dictar medidas de } \\
\text { protección a favor de la víctima. }\end{array}$ & 23 & 2,62 \\
\hline
\end{tabular}

De la tabla 04, se desprende que un porcentaje mayoritario de la muestra evidencia que los Jueces de Familia de la CSJT, durante el año 2017, han emitido medidas de protección de prohibición de incurrir en nuevos actos de violencia a efectos de que el agresor no vuelva a maltratar física, psíquica, sexual o patrimonialmente a la víctima. Además, se apercibe en las mismas resoluciones de medidas de protección de no hacer caso a este mandato de ser denunciado por el delito de Desobediencia y Resistencia a la Autoridad. Otra medida con gran prevalencia es la del impedimento de acercamiento o proximidad a la víctima, en donde la gran mayoría de resoluciones con esta medida se considera la distancia de 300 metros. Si bien es cierto la ley 30364 no señala la distancia especifica del acercamiento, pero los Jueces de Familia lo consideran así. Además de ello, algunos son más precisos señalando el no acercamiento, pero con fines de violencia, es decir permiten un acercamiento cuando tengan hijos en común, más por el contrario casi en la mayoría de resoluciones la distancia de $\mathbf{3 0 0}$ metros se conserva. También se considera ya en porcentajes minoritarios a las medidas de evaluación y tratamiento de víctima y agresor, esta medida con la finalidad de concurrir a seguir un tratamiento psicológico a efectos de mejorar la salud psicológica de agresor y víctima e hijos. Así también se tiene el retiro del agresor del domicilio, donde será la Policía Nacional a diferencia de la ley 26260 quien ejecute esta medida. También tenemos entre otras medidas 
requeridas para la protección de la integridad personal y la vida de sus víctimas o familiares a la visita social para efectos de conocer la situación socio familiar de la víctima y el agresor y como es el modo de vivencia con sus hijos, si mejoro o empeoro la situación de violencia denunciada con el tiempo. Así también se tiene, la prohibición de comunicación por vía telefónica, electrónica, chat, redes sociales, otras formas de comunicación, algunos jueces suelen precisar los números telefónicos que se prohíben llamadas a la víctima, y las redes sociales como Facebook, Twitter, y otras.

\section{DISCUSIÓN}

Se verificó que en el 97,34\% de las resoluciones judiciales no figura la condición de reincidencia en violencia familiar o de que se señale expresamente que se trata de una denuncia reiterada en contra del mismo agresor. Por otro, los resultados de la encuesta aplicada a magistrados, abogados defensores, abogados litigantes y representantes del Ministerio Publico, indican que una gran mayoría de los encuestados $77,13 \%$ señalan que el otorgamiento de medidas de protección por un juez de familia genera más eficacia que la dictada por un fiscal. Asimismo, una mayoría importante, $69,5 \%$ considera que el otorgamiento de medidas de protección por un juez genera más eficacia para no reincidir nuevamente en violencia familiar en la victima. Finalmente, el $60,12 \%$ señalan que el apercibimiento de ser denunciado por el delito de resistencia y desobediencia a la autoridad, si el agresor incumple con las medidas de protección dictadas, resulta eficaz para garantizar el derecho a la integridad sobre la víctima. Se corrobora con la revisión de resoluciones de medidas de protección que $93,2 \%$ se dictó a favor de la víctima medidas de protección fundadas, con lo que de alguna manera se salvaguarda su integridad. En conclusión, los resultados de la encuesta y el análisis de las resoluciones judiciales de otorgamiento de medidas de protección indican que las medidas de protección en procesos de violencia familiar son eficaces ya que garantizan el derecho a la integridad de las víctimas.

El artículo 23 de la ley 30364 (Ley para prevenir, sancionar y erradicar la violencia contra las mujeres y los integrantes del grupo familiar) señala expresamente: “(...) La Policía Nacional del Perú es responsable de ejecutar las medidas de protección dictadas, para lo cual debe tener un mapa gráfico y georreferencial de registro de todas las víctimas con las medidas de protección que les hayan sido notificadas; $y$, asimismo, habilitar un canal de comunicación para atender efectivamente sus pedidos de resguardo, pudiendo coordinar con los servicios de serenazgo a efectos de brindar una respuesta oportuna". El 52,79\% de los encuestados consideran que los mecanismos de supervisión de las medidas de protección por parte de la PNP si son eficaces. En consonancia a estos resultados, se tiene que el $52,79 \%$ de los encuestados refieren que si conoce que el cumplimiento de las medidas de protección se realiza a través de un seguimiento de manera periódica por parte de la Policía Nacional (principalmente en las Comisarias de Gregorio Albarracín Lanchipa.). Si bien la propia ley expresa taxativamente que es la Policía Nacional del Perú la entidad responsable de ejecutar las medidas de protección dictadas por el Juzgado de Familia correspondiente, la mayoría de los encuestados considera que existen mecanismos jurídicos de supervisión eficaces (fiscalizan y monitorean) los cuales están a cargo de la Policía Nacional y que permiten hacer un seguimiento de manera periódica. Las medidas de protección impuestas por los jueces en procesos de violencia familiar a las victimas disuaden considerablemente a los agresores a no reincidir en actos violentos.

El artículo 24 de la ley 30364 señala que: "El que desobedece, incumple o resiste una medida de protección dictada en un proceso originado por hechos que configuran actos de violencia contra las mujeres o contra integrantes del grupo familiar, comete delito de Resistencia o 
Desobediencia a la Autoridad previsto en el Código Penal". Debemos agregar que la inclusión de este artículo no garantiza necesariamente su cumplimiento, por lo que requiere ser contrastado en la realidad, para lo cual, se debe señalar que el $60,12 \%$ de los operadores de justica considera que el apercibimiento de ser denunciado por delitos de Resistencia y Desobediencia a la Autoridad si resulta eficaz para garantizar el derecho a la integridad de la víctima. Es decir, más de la mitad cree que la amenaza de una denuncia penal si frena de alguna manera que nuevas agresiones puedan repetirse en contra de la víctima de violencia familiar. Aunado a estos resultados se tiene que el $45,45 \%$ refiere que en la práctica conocen con poca frecuencia casos de reincidencia de violencia familiar denunciados por la víctima en contra del mismo agresor. Asimismo, el $12,32 \%$ señala que nunca conoce casos de reincidencia. Si se suman ambos, porcentajes, se obtiene un total de $57,77 \%$. Es decir, un poco más de la mitad de los encuestados considera que en la práctica judicial la reincidencia en casos de violencia familiar es poco frecuente o nula, con lo que sostenemos que la mejor demostración en la práctica es lo referido por los operadores del derecho, y ellos nos afirman que la amenaza de denuncias penales por el delito de Resistencia o Desobediencia a la Autoridad frena de alguna manera a los agresores para no reincidir nuevamente en actos violentos contra la víctima. Sin embargo, se debe tener en cuenta el bajo nivel de reincidencia encontrado en la presente investigación, lo cual es el 2,66\% de los expedientes judiciales donde se dictan medidas de protección, donde se observa que durante el año 2017 solo existió nueve casos de reincidencia. Las medidas de protección impuestas por los jueces en procesos de violencia familiar a las victimas disuaden considerablemente a los agresores a no reincidir en actos violentos.

La Comisión Provincial, debe difundir en forma amplia la ley 30364 para la prevención, sanción y erradicación de la violencia contra las mujeres y los integrantes el grupo familiar, ya que tiene entre sus funciones promover el cumplimiento de la ley. La Policía Nacional (Sección de Violencia Familiar) debe realizar un diseño de estrategia de seguimiento periódico de medidas de protección a las víctimas para que de esta manera se mejore los mecanismos de supervisión para la ejecución de medidas de protección, tal como lo viene realizando la Comisaria de Gregorio Albarracín así como habilitar un canal de comunicación directo entre autoridades y víctimas, existen encuestados que consideran que las medidas protección dictadas no viene siendo ejecutada óptimamente por parte de las autoridades policiales, por lo que se recomienda que la Policía Nacional coordine en forma más estrecha con los funcionarios de serenazgo tanto a nivel provincial como distrital para un proceso de seguimiento de las medidas de protección eficaz. La División de Medicina Legal de la región Tacna, debe implementar un número adecuado de psicólogos encargados de llevar a cabo las evaluaciones psicológicas ya que es insuficiente, y ello viene postergando hasta dos o tres meses de sucedidos los hechos denunciados, la evaluación psicológica a las víctimas. El Modulo de Familia, debe dictar directrices expresas a los Jueces de Familia a efectos que sean lo suficiente enfáticos en sus resoluciones donde emiten medidas de protección a las víctimas a efectos que los agresores no incurran en delito de Resistencia y Desobediencia a la Autoridad, ello debido a que el $39,88 \%$ de los operadores de justicia encuestados considere que este apercibimiento de ser denunciado por el referido delito ante el incumplimiento del agresor, no resulta eficaz para garantizar el derecho a la integridad sobre la víctima, nos lleva a recomendar lo siguiente: En primer lugar, muchos agresores no asisten a las audiencias de otorgamiento de medidas de protección y por ello, pueden no conocer los resultados de dichas audiencias, y por ende, el mandato judicial de no acercarse a la víctima, de no acosarla o intimidarla puede resultar infructuoso. Por todo ello, la eficacia del apercibimiento dependería de cual informado está el agresor y cuan consciente es de las consecuencias de su incumplimiento. 


\section{REFERENCIAS BIBLIOGRÁFICAS}

Acevedo, E. (2009). Separata de Derecho Constitucional II Parte I, Universidad Privada de Tacna. Perú.

Cifuentes, V. (2010). Análisis jurídico sobre la efectividad de las medidas de seguridad otorgadas a las mujeres víctimas de violencia intrafamiliar en el municipio de San Juan Sacatepéquez departamento de Guatemala. Tesis de la Universidad de San Carlos de Guatemala. Guatemala.

Chávarri. T. (2015). Influencia de la dependencia económica en la violencia conyugal de las mujeres agredidas atendidas en la DEMUNA del distrito de Yonan - provincia de Contumaza, 2015. Tesis de grado. Universidad de Trujillo.

Condori, M. (2016). Impacto de la ley 30364 en el centro emergencia mujer Ilave enero setiembre, 2016. Tesis. Universidad Andina Néstor Cáceres Velásquez de Puno.

Díaz, A. (2010). La efectividad de las medidas de protección frente a la violencia familiar. Tesis. Escuela de Post Grado de la Universidad Privada de Tacna.

El Peruano, Diario Oficial de Perú (octubre de 2016). Normas Legales: Guía de valoración del daño psíquico en personas adultas víctimas de violencia intencional. Lima. Recuperado de:

http://busquedas.elperuano.com.pe/normaslegales/anexo-de-la-res-n-3963-2016-mpfn-que-aprobo-guias-elabora-anexo-res-n-3963-2016-mp-fn-1444631-1/

García Belaunde, D. (1992). Esquema de la Constitución peruana. Lima: Ediciones Justo Valenzuela.

Hernández, G. (2014). El consentimiento de la víctima en los delitos de violencia intrafamiliar. Tesis para optar el grado de Magister en Derecho Penal en la Universidad de Sevilla (España).

INEI (2016). Brechas de Genero: Avances hacia la igualdad de mujeres y hombres. Lima: INEI.

Instituto Nacional de Estadística e Informática -INEI (2017). Encuesta Demográfica y de Salud Familiar-ENDES. 2016. Nacional y Regional. Recuperado de:

https://www.inei.gob.pe/media/MenuRecursivo/publicaciones_digitales/Est/Lib1433/ index.html

Junco, J. (2018). Separata de Estrategias de intervención legal y procedimiento de evaluación psicológica en casos de violencia contra la mujer y los integrantes del grupo familia ley 30364, Perú.

Loli, S. (2015). Violencia de género, marcos conceptuales. Lima: Separata taller de capacitación al personal del MIMP.

Lorente, M. (/2005). El agresor en la violencia de género: anatomía del maltratados, conferencia 25 de octubre del 2005, Gijón. España.

MIMP (2017), Plan Nacional de Igualdad de Género (2012-2017), Lima: Ministerio de la Mujer y Poblaciones Vulnerables. Recuperado de: https://www.mimp.gob.pe/files/direcciones/dgignd/planes/plan-nacional-igualdadgenero-2012-2017.pdf 
Morales, P. (2010). Lesiones y violencia de género frente a la jurisprudencia. Tesis fue sustentada para optar al Grado de Licenciado en Ciencias Jurídicas y Sociales en la Universidad de Chile.

Orna, O. (2013). Factores determinantes de la violencia familiar y sus implicancias -análisis de los estudios estadísticos sobre la violencia familiar en el distrito de San Juan de Lurigancho (Lima), Callao y otras ciudades del país. Tesis. Escuela de Post Grado de la Universidad Nacional Mayor de San Marcos. Perú.

Oxfam. Módulo I: Formación básica en género - Manual para participantes, La Paz, 2014. Recuperado de: https://peru.oxfam.org/sites/peru.oxfam.org/files/file_attachments/Manual \%20participantes\%20-\%20M\%C3\%B3dulo\%201\%20Formaci\%C3\%B3n\%20b \%C3\%A1sica\%20en\%20g\%C3\%A9nero_2.pdf

Rodríguez, B. (2013). Tratamiento de las medidas de protección dictadas por el ministerio público y su influencia en la violencia familiar entre cónyuges y convivientes en el distrito judicial de Tacna, periodo 2009-2010 Escuela de Posgrado de la UNJGB de Tacna.

Vera, M. (2014). Violencia intrafamiliar: las medidas de amparo y el principio de contradicción. Tesis optar el título de Magister en Derecho Civil y Procesal Civil en la Universidad Regional Autónoma de los Andes (Ecuador).

Ventura, B. (2016). El proceso por violencia familiar, como garantía de los derechos de las víctimas de violencia de género en el segundo juzgado de familia de Huánuco, 2014. Tesis. Universidad de Huánuco. Perú. 